\title{
Helmholtz Solitons: A New Angle in Nonlinear Optics
}

\author{
J. M. Christian, G. S. McDonald \\ Joule Physics Laboratory, School of Computing, Science and Engineering, Institute for Materials Research, \\ University of Salford, Salford M5 4WT, U.K. \\ P. Chamorro-Posada, J. Sánchez-Curto \\ Departmento de Teoría de la Señal y Comunicaciones e Ingeniería Telemática, Universidad de Valladolid, \\ ETSI Telecomunicación, Campus Miguel Delibes s/n, 47011 Valladolid, Spain
}

\begin{abstract}
New families of exact analytical Helmholtz solitons are presented for various material nonlinearities, and simulations confirm their stability. A novel generalization of Snell's Law for Kerr solitons is also reported. Helmholtz angular corrections to paraxial theory, exceeding 100\%, are uncovered. (C) 2007 Optical Society of America

OCIS codes: (190.4400) Nonlinear Optics, Materials; (190.9540) Nonlinear Optics; Self-Action Effects.
\end{abstract}

\section{Introduction}

We rigorously show that nonparaxial optical beams are not necessarily ultranarrow [1-3]. While existing paraxial models constrain considerations of broad beams to vanishingly-small propagation angles relative to the longitudinal (i.e. $z$ ) direction, arbitrary-angle (Helmholtz) effects yield geometrical corrections of arbitrary order and new qualitative phenomena. Conventional nonparaxial beam models (relying on perturbative expansions in terms of a single narrow-beam parameter) also prove to be invalid for describing regimes involving non-trivial angular geometries. Helmholtz corrections to paraxial theory can easily exceed 100\% (see Fig. 1) and are found to be essential for capturing oblique beam propagation, interaction, and interface effects [1]. A generalized Nonlinear Helmholtz (NLH) equation for the spatial part $E(x, z)$ of a broad TE-polarized electric field is [1-3]

$$
\left(\frac{\partial^{2}}{\partial z^{2}}+\frac{\partial^{2}}{\partial x^{2}}\right) E(x, z)+\frac{\omega^{2}}{c^{2}} n^{2}(E) E(x, z)=0 .
$$

Diffraction in NLH models is thus fully two-dimensional, occurring symmetrically in the $(x, z)$ coordinates which define the waveguide plane. Linear and nonlinear medium effects are described by the refractive-index function $n^{2}(E)$. Equation (1) possesses a large number of families of exact analytical solutions that are entirely physical [1-3].
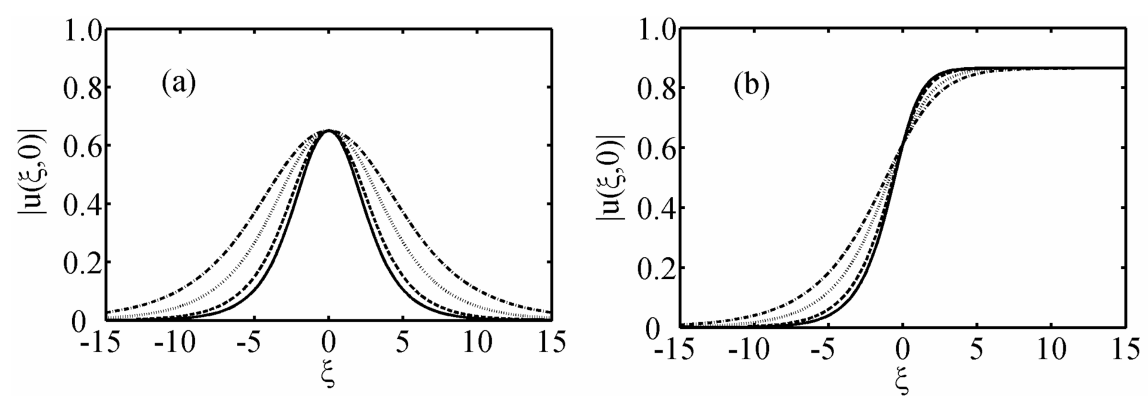

Fig. 1. Helmholtz geometrical corrections to the normalized beam profile for (a) hyperbolic and (b) boundary solitons of a cubic-quintic nonlinearity when propagating at an angle $\theta$ relative to the reference $z$ axis. Solid line: $\theta=0^{\circ}$ (paraxial profile); dashed line: $\theta=30^{\circ}$; dotted line: $\theta=50^{\circ}$; dot-dash line: $\theta=60^{\circ}$ (paraxial profile broadened by $100 \%$ ).

This presentation will draw on, and report significant extensions of, three of our most recent papers [1-3], in the area of Helmholtz soliton theory. A specific focus of novelty will be on new solution families arising from a wide range of material nonlinearities, such as cubic-quintic and saturable refractive indices. We will report the first exact analytical bistable Helmholtz solitons and demonstrate through simulations that the solitons lying on both branches of the bistable curve are robust attractors. We will also highlight novel types of solutions, such as the discovery of highly stable "Helmholtz boundary solitons" [see Fig. 1(b)]. Furthermore, for the general case of a dispersive $n^{2}(E)$, we will prove that the elliptic character of Eq. (1) is essential for describing arbitrary-angle effects. Ellipticity leaves in tact both the physical and the mathematical stability of exact solution families, as should be expected. 
Maintaining the numerical stability of these solutions also proves to be a straightforward task, and analysis has established that perceived instability in such elliptic models is a purely numerical artefact [2]. Finally, we have recently discovered [3], a compact version of Snell's Law for bright nonlinear beams that is valid for Kerr solitons refracting at an interface for arbitrary angles of incidence. We will report, for the first time, that this result extends consistently (in the same compact form) to dark Kerr solitons [4].

\section{Ultranarrow-beam vs. Helmholtz nonparaxiality}

The term "nonparaxial" is routinely used to refer to an optical beam whose waist $w_{0}$ is comparable to, or smaller than, its carrier wavelength $\lambda$. The condition $\lambda / w_{0} \equiv \varepsilon \sim O(1)$ then defines the so-called ultranarrow-beam scenario. To analyse such sub-wavelength fields, one is obliged to consider a perturbative expansion of Maxwell's equations in terms of the parameter-of-smallness $\varepsilon$ [5]. When $\varepsilon \sim O(1)$, nonlinear divergence can no longer be neglected and there may be strong coupling between the transverse and longitudinal components of the electric field. To $O\left(\varepsilon^{2}\right)$, the dominant transverse field component is governed by a Nonlinear Schrödinger (NLS) equation, supplemented by a range of higher-order nonlinear diffractive correction terms.

Helmholtz nonparaxiality is physically and mathematically distinct from ultranarrow-beam nonparaxiality. In Helmholtz soliton theory [1-4], optical beams are always assumed to be broad so that $\varepsilon<<O(1)$ is always fully satisfied. By omitting the slowly-varying envelope approximation, NLH models provide an excellent description of broad-beam evolution. Equation (1) possesses many types of exact analytical soliton solutions. Helmholtz nonparaxiality gives rise to a range of generic corrections to known paraxial solutions, and these corrections can exceed $100 \%$ (see Fig. 1). For example, the geometrical factor $2 \kappa V^{2}=\tan ^{2} \theta$, where $\kappa \sim \varepsilon^{2}, V$ is the soliton velocity (in a scaled coordinate system) and $\theta$ is the beam's propagation angle relative to the longitudinal ( $z$ ) axis, is a key feature of Helmholtz solutions. It depends solely upon the propagation angle $\theta$ of the beam and can be of any order, even when $\kappa<<(1)$. The origin of the $2 \kappa V^{2}$ correction lies in retaining the full generality of the $\partial^{2} / \partial z^{2}$ operator in Eq. (1), and it is thus independent of the system nonlinearity. Neither paraxial nor conventional narrow-beam models can access arbitrary-angle regimes since their governing equations lack the required $x-z$ symmetry.

\section{Hyperbolic vs. boundary solitons}

To facilitate a comparison with paraxial theory, Eq. (1) can be rewritten in a normalized form [1-4],

$$
\kappa \frac{\partial^{2} u}{\partial \zeta^{2}}+i \frac{\partial u}{\partial \zeta}+\frac{1}{2} \frac{\partial^{2} u}{\partial \xi^{2}}+f\left(|u|^{2}\right) u=0
$$

Here, $\zeta=z / L_{D}, \xi=2^{1 / 2} x / w_{0}$ and $L_{D}=k w_{0}{ }^{2} / 2$ is the diffraction length of a reference (paraxial) Gaussian beam of waist $w_{0}$. The (inverse) beam-width parameter is $\kappa=1 /\left(k w_{0}\right)^{2}=\varepsilon^{2} / 4 \pi^{2} n_{0}^{2}$. The wave-field $u$ is the normalized envelope of the complex electric field, $E(x, z)=E_{0} u(x, z) \exp (i k z)$, where $k=n_{0} k_{0}, k_{0}=2 \pi / \lambda$ and $n_{0}$ is the linear refractive index.

We have considered a range of refractive-index distributions $n^{2}(E)$, which are associated with the normalized nonlinearity functions $f\left(|u|^{2}\right)$, and have derived a number of exact analytical solutions. For example, a competing cubic-quintic nonlinearity $n(E)=n_{2} E^{2}-n_{4} E^{4}$, where $n_{2}>0$ and $n_{4}>0$, leads to $f\left(|u|^{2}\right)=\alpha|u|^{2}-\gamma|u|^{4}$. Equation (2) then permits the existence of hyperbolic soliton solutions. We have also recently derived a new class of boundary soliton solution [6],

where

$$
\begin{gathered}
u_{ \pm}(\xi, \zeta)=\left[\frac{\eta}{\exp [ \pm \Theta(\xi, \zeta)]+\Gamma}\right]^{\frac{1}{2}} \exp \left[i \sqrt{\frac{1+\kappa \mu^{2} / 2}{1+2 \kappa V^{2}}}\left(-V \xi+\frac{\zeta}{2 \kappa}\right)\right] \exp \left(-i \frac{\zeta}{2 \kappa}\right) \\
\Theta(\xi, \zeta)=\mu \frac{\xi+V \zeta}{\sqrt{1+2 \kappa V^{2}}}, \quad \eta=\frac{\mu^{2}}{2 \alpha} \Gamma, \quad \mu=\frac{\alpha}{2} \sqrt{\frac{6}{\gamma}} .
\end{gathered}
$$

Boundary solitons are of fundamental mathematical and physical interest. They expand the range of known exact analytical solutions of fully second-order non-integrable wave equations. Despite their spatially-extended structure, we have found that boundary solitons are remarkably stable against perturbations. These robust structures connect regions of finite- and zero-amplitude disturbance. This feature suggests that they also may be termed "edge solitons", since they can act as natural nonlinear boundary waves at the outer limits of, e.g. optical, disturbance. Moreover, the full Helmholtz framework of their definition permits the application of these exact analytical 
solutions with any orientation in the waveguide plane. Finite-size effects tend to play a profound role in twodimensional optical pattern formation and it seems plausible that our Helmholtz boundary solitons, or higherdimensional counterparts, may also find application in this important subject area.

\section{Snell's law: bright vs. dark Kerr solitons}

The behaviour of solitons at interfaces is an inherently angular problem, for which previous paraxial (i.e. NLS-type) analyses are restricted to bright solitons and vanishingly-small angles of incidence/reflection/refraction [7]. Helmholtz soliton theory has recently provided, for the first time, a framework for analysing the reflection and refraction properties of bright [1], and dark [4], solitons encountering an interface at arbitrary angles. Our study is based on an NLH model that is generalized to describe the evolution of solitons at the interface between two Kerr focusing (defocusing) media. Bright (dark) soliton solutions in each media are phase-matched at the interface, thus relating soliton transverse velocities in each medium. By exploiting the geometrical relation between velocities and propagation angles, we have discovered a remarkably compact Helmholtz generalization of Snell's law that governs Kerr soliton refraction:

$$
\gamma n_{01} \cos \left(\theta_{i}\right)=n_{02} \cos \left(\theta_{t}\right)
$$

In Eq. (4), $n_{01}$ and $n_{02}$ are the linear parts of the refractive index in each medium, $\theta_{i}$ and $\theta_{t}$ represent the angles of incidence and refraction with respect to the planar boundary separating the two media, and $\gamma$ is a nonlinear beam correction term. Although $\gamma$ assumes slightly different forms for bright and dark solitons, in both cases it exhibits a strong dependence on the Kerr coefficients of both media and the Helmholtz nonparaxial parameter $\kappa$.
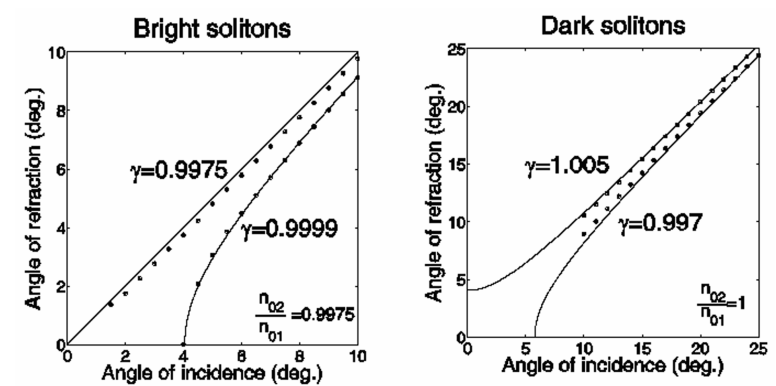

Fig. 2. Snell's law for Helmholtz bright (left) and dark (right) solitons. All angles shown are in the unscaled, i.e. the ( $x, z)$, coordinate frame. Numerical results from simulations (points) are compared with the theory (solid lines).

Excellent agreement has been found between analytical predictions (solid lines) and numerical simulations (points) for a wide range of interface parameters. For bright solitons, $\gamma$ can counteract the effect of the linear refractive-index mismatching, cancelling the critical angle and obtaining the interface transparency condition, $\theta_{i}=$ $\theta_{t}$. For dark solitons, $\gamma$ is shown able to determine the angle of refraction in absence of linear discontinuity at the interface, $n_{01}=n_{02}$. The compact generalization uncovered in our work, valid for different soliton types (bright and dark) and arbitrary angles, represents a new fundamental building block for studies involving the universal problem of soliton behaviour at the planar boundary of different nonlinear materials.

\section{References}

[1] P. Chamorro-Posada and G. S. McDonald, “Spatial Kerr soliton collisions at arbitrary angles,” Phys. Rev. E 74, 036609 (2006).

[2] J. M. Christian et al., "Helmholtz bright and boundary solitons," J. Phys. A: Math. \& Theor. 40, 1545-1560 (2007).

[3] J. Sánchez-Curto et al., "Helmholtz solitons at nonlinear interfaces," Opt. Lett. 32, 1126-1128 (2007).

[4] P. Chamorro-Posada and G. S. McDonald, "Helmholtz dark solitons," Opt. Lett. 28, 825-827 (2003).

[5] S. Chi and Q. Guo, "Vector theory of self-focusing of an optical beam in Kerr media," Opt. Lett. 20, 1598-1600 (1995).

[6] L. Gagnon, "Exact travelling-wave solutions for optical models based on the nonlinear cubic-quintic Schrödinger equation," JOSA A 9, 14771483 (1989).

[7] A. B. Aceves et. al.,"Theory of light-beam propagation at nonlinear interfaces. I. Equivalent-particle theory for a single interface," Phys. Rev. A 39, 1809-1827 (1989) 